\title{
SOME LIMITING DISTRIBUTIONS RELATED TO THE SUM OF A RANDOM NUMBER OF RANDOM VARIABLES 1
}

\author{
EDWARD PAULSON
}

1. Introduction. Let $X_{j}(j=1,2, \ldots)$ be a sequence of independent random variables with the same distribution, and consider the random variable $Y=X_{1}+X_{2}+\cdots+X_{N}$. The limiting distribution of $Y($ as $\lambda \rightarrow \infty)$ when $N$ is an integer-valued random variable whose distribution depends on a parameter $\lambda$ has been studied in detail by Robbins [1]. ${ }^{2}$ In this paper we shall attempt to extend some of Robbins' results by considering a more general type of statistic $Y_{1}$, which reduces to $Y$ in a special case. We shall also consider the limiting distribution of a second statistic $Y_{2}$, which reduces to the arithmetic mean $Y / N$ in a special case.

Where possible, we shall follow Robbins' notation. The probability that $N=k(k=0,1,2, \cdots)$ will be denoted by $\omega_{k}$, where the $\omega_{k}=\omega_{k}(\lambda)$ are functions of $\lambda$ so that, for all $\lambda, \omega_{k} \geqq 0$ and $\sum_{k=0}^{\infty} \omega_{k}=1$. Furthermore, we set

$$
\begin{aligned}
\alpha & =E(N)=\sum_{k=0}^{\infty} \omega_{k} \cdot k \\
y^{2} & =\operatorname{Var}(N)=\sum_{k=0}^{\infty} \omega_{k} \cdot(k-\alpha)^{2} \quad\left(y^{2}<\infty \text { for all } \lambda\right), \\
\theta(t) & =E\left[e^{i t((N-\alpha) / y)}\right]=\sum_{k=0}^{\infty} \omega_{k} \cdot e^{i t((k-\alpha) / y)} .
\end{aligned}
$$

Clearly $\alpha, y^{2}$, and $\theta(t)$ are functions of $\lambda$. We restrict ourselves in this paper to cases for which

$$
\operatorname{limit}_{\lambda \rightarrow \infty} \theta(t)=g(t)
$$

where $g(t)$ is a characteristic function, so that $(N-\alpha) / y$ has a limiting distribution as $\lambda \rightarrow \infty$.

2. The statistic $Y_{1}$. The statistic $Y_{1}$ to be considered is to have a distribution function $F(u)=P\left\{Y_{1} \leqq u\right\}$ of the form

$$
F(u)=\sum_{k=0}^{\infty} \omega_{k} \cdot F_{k}(u)
$$

where $F_{k}(u)$ denotes the conditional distribution of $Y_{1}$ when $N=k$;

Received by the editors April 15, 1949 and, in revised form, July 23, 1949.

${ }^{1}$ Work done under the sponsorship of the Office of Naval Research.

2 Numbers in brackets refer to the references cited at the end of the paper. 
this conditional distribution $F_{k}(u)$ shall be subject to the essential restriction that there exist constants $a$ and $c$ (with $c>0$ ) so that

$$
\lim _{k \rightarrow \infty} \int_{-\infty}^{\infty} e^{i \ell\left((u-a k) / c k^{1 / 2}\right)} d F_{k}(u)=q(t)
$$

where $q(t)$ is another characteristic function. It is clear that $Y_{1}$ will reduce to $Y$ when $F_{k}(u)$ is the $k$ th convolution with itself of a random variable $X$ such that $E(X)=a, E(X-a)^{2}=c^{2}, 0<c^{2}<\infty$.

Now let $\sigma^{2}=\alpha c^{2}+y^{2} a^{2}$, and consider the random variable

$$
Z_{1}=\frac{Y_{1}-a \alpha}{\sigma} .
$$

Theorem 1. Suppose $a \neq 0$. If as $\lambda \rightarrow \infty$ limit $\alpha=\infty$, limit $\alpha / y^{2}=m$ $(0<m<\infty)$, then $Z_{1}$ has a limiting distribution whose characteristic function $\Phi(t)$ is given by

$$
\Phi(t)=g\left(t\left(\frac{a^{2}}{a^{2}+c^{2} m}\right)^{1 / 2}\right) \cdot q\left(t\left(\frac{c^{2} m}{a^{2}+c^{2} m}\right)^{1 / 2}\right) .
$$

The proof consists in showing that limit $\lambda_{\lambda \rightarrow \infty} E\left(e^{i t Z_{1}}\right)=\Phi(t)$ for every real $t$. In the proof we shall use $o(1)$ to denote a quantity which $\rightarrow 0$ as $\lambda \rightarrow \infty$, and $o^{*}(1)$ to denote a quantity which $\rightarrow 0$ as $k \rightarrow \infty$.

We have

$$
E\left(e^{\left.i t Z_{1}\right)}=\sum_{k=0}^{\infty} \omega_{k} \cdot \int_{-\infty}^{\infty} e^{i t((u-a \alpha) / \sigma)} d F_{k}(u) .\right.
$$

Since the hypotheses of Theorem 1 imply $y \rightarrow \infty$ as $\lambda \rightarrow \infty$, we find that $P\left\{|(N-\alpha) / y|>y^{1 / 2}\right\}=o(1)$ from Tchebycheff's inequality. Hence

$$
\begin{aligned}
E\left(e^{i t Z_{1}}\right) & =o(1)+\sum_{|k-\alpha| \leqq y^{3 / 2}} \omega_{k} \cdot \int_{-\infty}^{\infty} e^{i t((u-\alpha \alpha) / \sigma)} d F_{k}(u) \\
& =o(1)+\sum_{|k-\alpha| \leq y^{3 / 2}} \omega_{k} e^{i a t((k-\alpha) / \sigma)} \int_{-\infty}^{\infty} e^{i t^{\prime}\left((u-a k) / c k^{1 / 2}\right)} d F_{k}(u) \\
& \text { where } t^{\prime}=t\left(\frac{c k^{1 / 2}}{\sigma}\right) .
\end{aligned}
$$

Since $t\left(c k^{1 / 2} / \sigma\right)=t\left(c^{2} m /\left(c^{2} m+a^{2}\right)\right)^{1 / 2}+o(1)$ for $|k-\alpha| \leqq y^{3 / 2}$, we see that $t^{\prime}$ lies in a finite interval for any fixed value of $t$. Since (1) implies uniform convergence in any finite interval, we have uniformly for all $k$ such that $|k-\alpha| \leqq y^{8 / 2}$ 


$$
\begin{aligned}
\int_{-\infty}^{\infty} e^{i t^{\prime}\left((u-a k) / c c^{1 / 2}\right)} d F_{k}(u) & =q\left(t^{\prime}\right)+o^{*}(1) \\
& =q\left(t^{\prime}\right)+o(1) \\
& =q\left(i\left(\frac{c^{2} m}{c^{2} m+a^{2}}\right)^{1 / 2}\right)+o(1),
\end{aligned}
$$

making use of the fact that $\alpha-y^{3 / 2} \rightarrow \infty$ as $\lambda \rightarrow \infty$, and that a characteristic function is everywhere continuous. Combining these results we obtain

$$
E\left(e^{i t Z 1}\right)=o(1)+q\left(t\left(\frac{c^{2} m}{c^{2} m+a^{2}}\right)^{1 / 2}\right) \sum_{|k-\alpha| \leqq y^{3 / 2}} \omega_{k} \cdot e^{i a t((k-\alpha) / \sigma)} .
$$

Now

$$
\begin{aligned}
\sum_{|k-\alpha| \leq y^{0 / 2}} \omega_{k} \cdot e^{i a t((k-\alpha) / \sigma)} & =o(1)+\sum_{k=0}^{\infty} \omega_{k} \cdot e^{i a t(y / \sigma)((k-\alpha) / y)} \\
& =o(1)+\left[o(1)+g\left(t \frac{a y}{\sigma}\right)\right] \\
& =o(1)+g\left(t\left(\frac{a^{2}}{c^{2} m+a^{2}}\right)^{1 / 2}\right) .
\end{aligned}
$$

We now have

$$
E\left(e^{i t Z_{1}}\right)=o(1)+g\left(t\left(\frac{a^{2}}{c^{2} m+a^{2}}\right)^{1 / 2}\right) \cdot q\left(t\left(\frac{c^{2} m}{c^{2} m+a^{2}}\right)^{1 / 2}\right),
$$

which proves Theorem 1 .

By proceeding in a similar manner, it is easy to verify that if either of the two sets of conditions (1) $a \neq 0$, limit $\alpha=\infty$, limit $\alpha / y^{2}$ $=\infty$, or (2) $a=0$, limit $\alpha=\infty$, limit $\alpha / y^{2}=m^{\prime}\left(0<m^{\prime} \leqq \infty\right)$ hold, then $Z_{1}$ has a limiting distribution with characteristic function $q(t)$.

3. The statistic $Y_{2}$. The statistic $Y_{2}$ again has a distribution function $F(u)=P\left\{Y_{2} \leqq u\right\}$ of the form

$$
F(u)=\sum_{k=0}^{\infty} \omega_{k} \cdot F_{k}(u)
$$

but the conditional distribution $F_{k}(u)$ of $Y_{2}$ when $N=k$ is now subject to the restriction that there exist a constant $a$ such that

$$
\lim _{k \rightarrow \infty} \int_{-\infty}^{\infty} e^{i t k^{1 / 2}(u-a)} d F_{k}(u)=m(t)
$$


where $m(t)$ is a characteristic function.

Now let $Z_{2}=\alpha^{1 / 2}\left(Y_{2}-a\right)$.

THeorem 2. If as $\lambda \rightarrow \infty$ limit $\alpha=\infty$, limit $\alpha / y^{2}=m^{\prime}\left(0<m^{\prime} \leqq \infty\right)$, then $Z_{2}$ has a limiting distribution corresponding to the characteristic function $m(t)$.

The proof again consists in showing that limit $\lambda_{\lambda \rightarrow \infty} E\left(e^{i t Z_{2}}\right)=m(t)$.

$$
\begin{aligned}
E\left(e^{i t Z_{2}}\right) & =\sum_{k=0}^{\infty} \omega_{k} \cdot \int_{-\infty}^{\infty} e^{i t \alpha^{1 / 2}(u-\alpha)} d F_{k}(u) \\
& =o(1)+\sum_{|k-\alpha| \leqq y^{3 / 2}} \omega_{k} \cdot \int_{-\infty}^{\infty} e^{i t(\alpha / k)^{1 / 2}\left[k^{1 / 2}(u-\alpha)\right]} d F_{k}(u) \\
& =o(1)+\sum_{|k-\alpha| \leqq y^{3 / 2}} \omega_{k}\left[m\left(t\left(\frac{\alpha}{k}\right)^{1 / 2}\right)+o(1)\right] \\
& =o(1)+m(t) \sum_{|k-\alpha| \leqq y^{3 / 2}} \omega_{k} \\
& =o(1)+m(t),
\end{aligned}
$$

which proves Theorem 2 .

4. Some examples. We conclude this paper by giving an example involving Theorem 1, and an example involving Theorem 2. First let $N$ have a Poisson distribution with mean $\lambda$, so that $\omega_{k}=e^{-\lambda} \lambda^{k} / k$ !, $\alpha=\lambda, y^{2}=\lambda$. Let $\bar{x}=(1 / N) \sum_{i=1}^{N} x_{i}$ and put $Y_{1}=\sum_{i=1}^{N}\left(x_{i}-\bar{x}\right)^{2}$ where $\left\{x_{i}\right\}(i=1,2, \cdots)$ is a sequence of independent observations on a random variable whose fourth central moment $\mu_{4}$ is finite. If we make use of the known fact that the sample variance (based on $k$ observations) is asymptotically normal with mean $\mu_{2}$ and variance $\left(\mu_{4}-\mu_{2}^{2}\right) / k$, it follows from Theorem 1 that as $\lambda \rightarrow \infty$ the limiting distribution of $\left(Y_{1}-\lambda \mu_{2}\right) /\left(\lambda \mu_{4}\right)^{1 / 2}$ is normal with zero mean and unit variance.

For the second example, let $N$ have a binomial distribution with parameters $\lambda, p, q=1-p$, so that $\omega_{k}=C_{\lambda, k} p^{k} q^{\lambda-k}(k=0,1,2, \cdots, \lambda)$. Now let $Y_{2}=r / N$ where $r$ is the number of successes in $N$ independent trials with constant probability $P$ of a success. It follows from Theorem 2 that $(\lambda p)^{1 / 2}\left[Y_{2}-P\right]$ has in the limit a normal distribution with zero mean and variance $P(1-P)$. The statistic in the second example arose in a problem considered by Birnbaum and Sirken where $Y_{2}$ represented the percentage of people voting yes when only $N$ out of a random sample of $\lambda$ were available for interviewing. The limiting distribution of $Y_{2}$ for this special case was obtained by Birnbaum and Sirken by a different method. 


\section{REFERENCES}

1. H. Robbins, The asymptotic distribution of the sum of a random number of random variables, Bull. Amer. Math. Soc. vol. 54 (1948) pp. 1151-1162.

2. H. Cramer, Mathematical methods of statistics, Princeton University Press, 1946, p. 365 .

3. Z. W. Birnbaum and M. Sirken, Bias due to non-availability in sampling surveys, Journal of the American Statistical Association vol. 45 (1950) pp. 98-111.

UNIVERSITY OF MICHIGAN

\section{BOUNDS FOR THE COEFFICIENTS OF UNIVALENT FUNCTIONS}

ARYEH DVORETZKY

1. The purpose of this note is to give some estimates for the moduli of the coefficients $a_{n}$ of

$$
w=f(z)=z+a_{2} z^{2}+\cdots+a_{n} z^{n}+\cdots,
$$

assumed regular and univalent in $|z|<1$, in terms of the domain onto which $|z|<1$ is mapped through (1). A typical result, cf. (27), is that if this domain does not cover arbitrarily large circles, then ${ }^{1}$ $a_{n}=O(\log n)$.

Let $W$ be the domain in the $w$-plane onto which $|z|<1$ is mapped through (1) and denote by $A(R)$ the radius of the largest circle with center on $|w|=R$ the whole interior of which is contained in $W$, that is,

$$
A(R)=\max _{|w|=R} \min _{w^{\prime} \notin W}\left|w^{\prime}-w\right| \quad(0 \leqq R<\infty) .
$$

Our aim is to derive upper bounds for $\left|a_{n}\right|$ in terms of $A(R)$.

Always $A(R) \leqq R+1$ while $A(R)=0$ for sufficiently large $R$ if and only if (1) is bounded in $|z|<1$. The function $A(R)$ measures, in some sense, the extension of $W$. This "extension" has, however, little to do with area, in fact whatever the positive function $a(R)$ there exists a function (1) mapping $|z|<1$ on a slit domain and for which $A(R)=o(a(R))$.

Presented to the Society, August 30, 1949; received by the editors March 31, 1949 and, in revised form, July 6, 1949.

1 Throughout the paper, $O$ and $o$ refer to $n \rightarrow \infty$ or $R \rightarrow \infty$ according to which of the variables $n$ or $R$ appears in the formula. 